\title{
La Pathologie Anorectale Au Chu Campus De Lome (Togo)
}

\author{
A. Bagny \\ LM. Lawson-Ananissoh \\ O. Bouglouga \\ YR. EI Hadji \\ LY. Kaaga \\ D. Redah
}

Service d’hépato-gastroentérologie, CHU Campus de Lomé

MA. Djibril

Service de Médecine interne, CHU Sylvanus Olympio

doi: 10.19044/esj.2016.v13n3p423 URL:http://dx.doi.org/10.19044/esj.2016.v13n3p423

\begin{abstract}
Objective: To describe the clinical and endoscopic presentation of anorectal pathologies in the gastroenterology department of the CHU campus of Lomé. Patients and methods: Retrospective study based on reports of low digestive endoscopy carried out at the university teaching hospital Campus of Lome. We collected the age of the patients, their sex, the indications of the examination and the different anorectal lesions encountered. Results: The mean age was $41.8 \pm 14.3$ years. There was a predominance of $30-40$ years (38.8\%). There was also a male predominance with a sex ratio of 1.6. The most frequent indication was rectal bleeding (64.1\%). Anal pain was found in $40.8 \%$ of cases. The most frequent lesions were hemorrhoids (69.9\%) and anal fistula in $8.7 \%$ of cases. Cancer of the anus was rarely observed (1.9\%) as was the rectal polyp (0.1\%). Conclusion: Anorectal pathologies are relatively frequent in our department, especially in young patients and are dominated by hemorrhoidal disease.
\end{abstract}

Keywords: Hemorrhoid, anal cancer, rectorrhagia, Togo

\section{Resume}

Objectif : décrire la présentation clinique et endoscopique des pathologies anorectales dans le service de gastroentérologie du CHU campus de Lomé. Patients et méthodes : Étude rétrospective à partir des comptesrendus d'endoscopie digestive basse réalisée au CHU Campus de Lomé. 
Nous avons recueilli l’âge des patients, leur sexe, les indications de l'examen et les différentes lésions anales et rectales rencontrées. Résultats : L’âge moyen était de 41.8土14,3 ans avec des extrêmes de 17 et 83 ans. On notait une prédominance des 30-40 ans (38,8\%).On notait également une prédominance masculine avec un sex-ratio (H/F) de 1,6. L’indication la plus fréquente était la rectorragie (64,1\%). Les douleurs anales ont été retrouvées dans 40,8\% des cas. Les lésions les plus fréquentes étaient les hémorroïdes (69,9\%) puis la fistule anale dans 8,7\% des cas. Le cancer de l'anus a été rarement observé $(1,9 \%)$ de même que le polype rectal $(0,1 \%)$. Conclusion : Les pathologies anorectales sont relativement fréquentes dans notre service, surtout chez les patients jeunes et sont dominées par la maladie hémorroïdaire.

Mots clés: Hémorroïde, cancer de l’anus, rectorragie, Togo

\section{INTRODUCTION}

La pathologie ano-rectale demeure un motif fréquent de consultation aussi bien en médecine générale qu'en gastroentérologie (Pfenninger JL et al, 2001 ; Tade AO et al, 2004). Elle est probablement sous-estimée en Afrique noire du fait de la pudeur, du recours à la médecine traditionnelle, de la négligence et du manque d'informations (Darie $\mathrm{H}$ et al, 1996). Plusieurs études ont été consacrées à la pathologie ano-rectale en Afrique tropicale (Dia D et al, 2010- Okon JB et al 2012) mais aucune n’a été réalisée dans notre pays et particulier dans notre service jusqu’à présent. Les objectifs de notre étude étaient de décrire la présentation clinique et endoscopique des pathologies ano-rectales dans le service de gastroentérologie du CHU campus de Lomé.

\section{PATIENTS ET METHODES}

Il s’agissait d'une étude rétrospective à partir des comptes-rendus d'endoscopies digestives basses réalisées au CHU Campus de Lomé durant la période du 2 janvier au 31 décembre 2015. Tous les comptes-rendus d’anorectoscopie interprétables ont été pris en compte. L'examen anorectoscopique se faisait sans prémédication. Un simple lavement rectal à base de laxatif était fait le jour même de l'examen. Nous avons recueilli l'âge des patients, leur sexe, les indications de l'examen et les différentes lésions anales et rectales rencontrées.

\section{RESULTATS}

Durant la période d'étude 103 ano-rectoscopies ont été effectuées sur 436 endoscopies basses soit 23,6\%. Sur les 103 patients, 64 étaient de sexe masculin et 39 de sexe féminin soit un sex-ratio (H/F) de 1,6. 


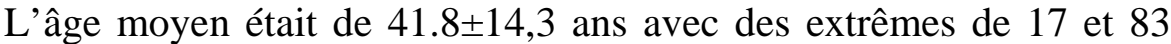
ans. On notait une prédominance des 30-40 ans (38,8\%). L'indication la plus fréquente était la rectorragie (64,1\%). Les douleurs anales ont été retrouvées dans 40,8\% des cas (Tableau 1). La lésion la plus fréquente a été les hémorroïdes (69,9\%) ensuite la fistule anale dans 8,7\% des cas (Tableau 2). Le cancer du canal anal a été rarement observé (1,9\%) de même que le polype rectal $(0,1 \%)$.

Les patients atteint de la maladie hémorroïdaire avaient un âge moyen de 41ans avec une prédominance masculine (18 hommes pour 2 femmes), les indications dans ce cas étaient la rectorragie (80\%), la proctalgie (40\%), puis le prolapsus, l'écoulement anal et la tuméfaction anale dans respectivement $5 \%$ des cas chacun. Les hémorroïdes étaient à localisation interne (38,8\%) et ou externe (31,1\%). L'association des indications ou des lésions après examen proctologique était possible chez un même patient. Il existait une thrombose hémorroïdaire dans 13,04\% des cas.

Les patients porteurs de fissure anale avaient un âge moyen de 28,6 ans, et un sex-ratio de 0,6. L'examen était indiqué par les saignements minimes (80\%) et la proctalgie (60\%) et le syndrome fissuraire (40\%). Ces fissures anales étaient antérieures dans $100 \%$ des cas dans notre étude et jeunes (60\%).

Les patients porteurs de fistule anale avaient un âge moyen de 41,8ans. Avec un sex-ratio de 0,6. Les indications de l'anorectoscopie étaient la proctalgie $(71,4 \%)$ et la suppuration anale $(28,6 \%)$.

Le cancer du canal anal était diagnostiqué chez 2 patients de sexe masculin, chez qui l'examen était indiqué par la rectorragie et la proctalgie. Ces patients avaient consulté tardivement parce qu'ils pensaient êtres porteurs d'une maladie hémorroïdaire. Dans les 2 cas il s’agissait de tumeur ulcéro-bourgeonnante et stenosante. L’histologie avait révélé dans les deux cas (100\%) qu’il s’agissait d’un cancer épidermoïde.

\section{DISCUSSION}

Les études sur les pathologies anorectales sont rares en Afrique noire (Dia D et al, 2010). La plupart de ces études (Dia D et al, 2010 - Okon JB et al ,2012) étaient basées sur les données endoscopiques à l’instar de notre étude. Notre étude a cependant pour avantage de décrire l'épidémiologie et de faire le panorama des pathologies anorectales dans notre service. L’âge moyen était de 41.8 $\pm 14,3$ ans comparable à d'autres séries africaines (Mahassadi KA et al ,2012 ; Ndjitoyap Ndam EC et al 1991 ; N'dri N et al, 1994). Nous avions retrouvé une prédominance masculine (sex-ratio $=1,6$ ) comparable à la série de Nditoyap et al (1991), mais nettement inferieure à celle de Ndri et al (1994) en Côte d’ivoire qui avaient trouvé 3,6. Dans notre étude comme dans la plus part des séries africaines (Dia D et al, 2010 - 
Ankouane F et al, 2015) la rectorragie était l’indication la plus fréquente. Ceci pourrait être lié à l'anxiété et le stress causé par la vue du sang, en particulier chez des sujets africains jeunes de sexe masculin pour qui le saignement par voie basse constitue un phénomène pouvant entacher l'intégrité de leur virilité. De plus le saignement par voix haute ou basse dans notre conception africaine est un signe de fatalité et de mort imminente (Bagny A et al, 2010).

La maladie hémorroïdaire était la pathologie ano-rectale la plus rencontrée dans notre étude comme dans d'autres études africaines (Darie $\mathrm{H}$ et al, 1996 ; Dia D et al, 2010 ; Maiga MY et al, 1995) ; dans notre étude comme dans celles d'autres auteurs africains suscités, les hémorroïdes étaient retrouver surtout chez les sujets jeunes de sexe masculin. Ces hémorrö̈des étaient source de saignement et de douleur anale, qui motivaient parfois des traitements traditionnels souvent responsables de complications graves à types de surinfection ou d'ulcération (Bougouma A et al, 2012). La fistule anale était la deuxième pathologie fréquente dans notre étude $(8,7 \%)$ ce résultat est comparable à celui de Dia et al (2010); mais habituellement la prévalence des fistules anales est faibles dans la plupart des études africaines (Ndjitoyap Ndam E C et al 1991 ; Maiga MY et al, 1995 ; Yassibanda S et al, 2004) probablement à cause de la pudeur ou par simple négligence. Le fort pourcentage de ces fistules dans notre série pouvait s'expliquer par le fait qu'il s'agissait de sujet jeunes qui présentaient souvent une proctalgie et ou une suppuration.

La fissure anale qui représente la deuxième plus fréquente pathologie ano-rectale (Tade AO et al, 2004 ; Dia D et al, 2010 ; Ndjitoyap Ndam EC et al 1991) était cependant moins fréquente dans notre service comme dans celle de Yassibanda S et al (2004) à Bangui. Ce faible pourcentage pourrait aussi être le faite de la pudeur, la négligence ou le manque d'information.

Dans notre étude la pathologie tumorale était représentée par les cancers ano-rectaux et les polypes. Ces données étaient supérieures aux données de la littérature (Dia D et al, 2010 ; Yassibanda S et al 2004). Ce résultat pourrait s'expliquer par le fait que notre service soit le seul service de référence publique dans la ville de Lomé. Ces tumeurs étaient observés à des stades tardifs, car souvent les patients essayaient d'abord d'autres types de traitements, notamment traditionnel et ne consultaient que lorsque les symptômes persistaient ou s'aggravaient malgré le traitement traditionnel. Ce constat pose le problème de sensibilisation et d'information de nos populations en milieu tropical sur les pathologies ano-rectales en général et tumorale en particulier. Car s'agissant de la pathologie tumorale ano-rectale, un simple toucher rectal peut permettre d'évoquer le diagnostic. 


\section{CONCLUSION}

Les pathologies anorectales sont relativement fréquentes dans notre service, elles sont fréquentes chez les patients jeunes et sont dominées par la maladie hémorroïdaire. La prévalence des cancers ano-rectaux n’est pas négligeable et devrait faire l'objet de plus d'information en particulier dans le contexte où il peut être facilement confondu a une maladie hémorroïdaire.

\section{References:}

1. PFENNINGER JL, ZAINEA GC. (2001) Common anorectal conditions : Part II. Lésions. Am Fam Physician ; 64 (1) : 77-88.

2. TADE AO, SALAMI BA, MUSA AA, ADENIJI AO. (2004) Anal complaints in Nigerians attending Olabisi Onabanjo University Teaching Hospital (OOUTH), Sagamu. Niger Postgrad Med J ; 11 (3) :218-20.

3. DARIE H, KLOTZ F (1996). La pathologie anale et péri-anale en zone tropicale. Acta endoscopica ; 26, 9- 16.

4. DIA D, DIOUF ML, MBENGUE M, BASSENE ML, FALL S, DIALLO S et al. (2010) Pathologies anorectales à Dakar, analyse de 2016 examens proctologiques. Med Afr Noire ; 57 :241-244

5. MAHASSADI KA, SORO KG, KOUAKOU B, ANZOUA KI, BANGOURA DA. (2012) Les affections colo-proctologiques et leurs déterminants au CHU de Yopougon (Côte d'Ivoire). Rev int sc méd; 14: 73-77.

6. OKON JB, NDRI N, TOTH'O A, ASSI C, DIAKITE M, SORO D et al. (2012) Diagnostic des rectorragie au centre hospitalier universitaire de Cocody à Abidjan (Côte d'ivoire). Médecine et sante tropicale ; 22 :398-400

7. NDJITOYAP NDAM E C, NJOYA O, MBALLA E, NSANGOU MF, NJAPOM C, MOUKOURI NYOLO E et al. (1991) Apport de l'endoscopie dans la pathologie digestive basse en milieu Camerounais, étude analytique de 720 examens. Med Afr Noire, 38 (12) :835-841

8. N'DRI N, KOUAKOU LOHOUES MJ, ATTIA KA, MOUSTAPHA OM, YASSIBANDA S, BOUGOUMA A, et al. (1994) La maladie hémorroïdaire en milieu africain : à propos de 552 cas colligés au CHU de Cocody. Med Chir Dig ; 23 : 233-4

9. ANKOUANE F, KOWO M, BIWOLE SIDA M, TZEUTON C, NDJITOYAP NDAM EC. (2015) Anus Diseases in Proctology Consultation in the Yaounde University Teaching Hospital (Cameroon): Male Predominance, Taboo and Neglected Diseases. Austin J Gastroenterol ; 2(5): 1051. 
10. BAGNY A, BOUGLOUGA O, DJIBRIL MA, DUSABE A, MBA KB,KAAGA LY et al (2010) Aspects psychopathologiques au cours des hématémèses au Togo : représentation et vécu chez les patients. J. Afr. Hépatol. Gastroentérol ; 4:216-219

11. MAÏGA MY, TRAORE HA, DIALLO G, DEMBELE K, KALLE A, DEMBELE M, GUINDO A.(1995) Etude épidémiologique de la pathologie anale au Mali. Med Chir Dig ; 24 : 269-70.

12. BOUGOUMA A, GIUNGANE NA, SOMBIE RA.(2012) La pathologie anorectale en milieu hospitalier à Ouagadougou (approche endoscopique): aspects épidémiologiques et diagnostiques. Med Afr Noire; 5: 87-94.

13. MAIGA MY, TRAORE HA, DIALLO G, DEMBELE M, DEMBELE K, KALLE A, GUINDO A. (1995) Aspects cliniques et anatomopathologiques du cancer du canal anal au Mali : à propos de 9 cas. Méd Chir Dig ;24 : 423-424.

14. YASSIBANDA S, IGNALEAMOKO A, MBELESSO P, BOBOSSI GS, BOUA N, CAMEOGO-POLICE SM et al. (2004) La pathologie ano-rectale à Bangui République de centre Afrique. Mali Med; 19(2) :12-14

Tableau 1 : indications de l'endoscopie basse

\begin{tabular}{|l|c|c|}
\hline & Effectif (n) & Pourcentage (\%) \\
\hline Rectorragie & 66 & 64,1 \\
\hline Proctalgie & 42 & 40,8 \\
\hline Sensation de masse & 30 & 29,1 \\
\hline Ecoulement anal & 06 & 5,8 \\
\hline Bilan d'extension cancer du col de l'utérus & 06 & 5,8 \\
\hline Constipation & 04 & 3.8 \\
\hline Prolapsus & 03 & 2,9 \\
\hline Brûlure anale & 03 & 2,9 \\
\hline
\end{tabular}

Tableau 2: Répartition des lésions

\begin{tabular}{|l|c|c|}
\hline & Effectif (n) & Pourcentage (\%) \\
\hline Absence de lésion & 38 & 38,8 \\
\hline Hémorroïde interne & 38 & 38,8 \\
\hline Hémorroïde externe & 32 & 31,1 \\
\hline Fistule anale & 9 & 8,7 \\
\hline Ano-rectite & 8 & 7,7 \\
\hline Cancer rectal & 6 & 5,8 \\
\hline Fissure anale & 5 & 4,8 \\
\hline Abcès anal & 4 & 3,8 \\
\hline Mycose péri-anale & 4 & 3,8 \\
\hline Rectocolite ulcéro-hémorragique & 3 & 2,9 \\
\hline Cancer de l'anus & 2 & 1,9 \\
\hline Polype rectal & 1 & 0,1 \\
\hline
\end{tabular}

